\title{
RESEARCH
}

Open Access

\section{Aerobic and resistance training enhances endothelial progenitor cell function via upregulation of caveolin-1 in mice with type 2 diabetes}

Lu Zhai', Yuhua Liu', Wenpiao Zhao², Qingyun Chen', Tao Guo³, Wei Wei', Zhuchun Luo ${ }^{5,1}$, Yanfeng Huang ${ }^{1}$, Cui $\mathrm{Ma}^{1}$, Feng Huang ${ }^{3^{*}}$ and Xia Dai ${ }^{\text {* }^{*}}$

\begin{abstract}
Background: To explore the effect of aerobic training (AT), resistance training (RT) or a combination of AT and RT $(A T+R T)$ on the function of endothelial progenitor cells (EPCS) in mice with type 2 diabetes and the potential effective mechanisms

Methods: Eight-week-old db/db male mice were used as type 2 diabetic animal models in this study. Mice were randomly assigned to the control group $(n=5)$, AT group $(n=5)$, RT group $(n=5)$ and AT+RT group $(n=5)$. Mice in the control group remained sedentary with no specific training requirement. Mice were motivated to perform AT, RT or AT+RT by a gentle pat on their body for 3 or 4 days/week for 14 days. AT was performed by treadmill running, RT was performed by ladder climbing and AT+RT involved both AT and RT. Bone-derived EPCs were isolated after 14 days of the intervention. EPC expression of CD31, CD34, CD133, CD144 and VEGFR2 was detected by immunofluorescence staining. Fluorescence detection was performed on attached mononuclear cells to detect double-positive EPCs. We then explored the effect of caveolin-1 knockdown (lentiviral vector with caveolin-1-siRNA) on the proliferation and adherence of EPCs and the concentration of caveolin-1 and PI3K AKT via western blot analyses.
\end{abstract}

Results: Compared to the mice in the control group, the mice in the AT, RT and AT+RT groups presented significant increases in proliferation and adherence after 14 days of intervention. AT+RT induced an increase in EPC adherence, which was greater than that of the control, RT and AT groups. Caveolin-1 knockdown inhibited the EPC proliferative and adherent abilities. The AT+RT group showed higher levels of caveolin-1 and p-AKT than the control group, but these changes were decreased by caveolin-1-siRNA transfection.

Conclusion: Combined AT and RT is an effective way to improve EPC function through upregulation of caveolin-1 in mice with type 2 diabetes.

Keywords: Endothelial progenitor cell, Type 2 diabetes, Aerobic and resistance training, Caveolin-1

\footnotetext{
*Correspondence: huangfeng3000@126.com; daixia1396323@163.com

${ }^{3}$ Department of Cardiology, The First Affiliated Hospital of Guangxi Medical University, Nanning 530021, China

'Department of Endocrinology, The First Affiliated Hospital of Guangxi

Medical University, Nanning 530021, China

Full list of author information is available at the end of the article
}

(c) The Author(s). 2020 Open Access This article is distributed under the terms of the Creative Commons Attribution 4.0 International License (http://creativecommons.org/licenses/by/4.0/), which permits unrestricted use, distribution, and reproduction in any medium, provided you give appropriate credit to the original author(s) and the source, provide a link to the Creative Commons license, and indicate if changes were made. The Creative Commons Public Domain Dedication waiver (http://creativecommons.org/publicdomain/zero/1.0/) applies to the data made available in this article, unless otherwise stated. 


\section{Background}

Bone marrow-derived endothelial progenitor cells (EPCs) can self-proliferate to increase their cell number and adhere to injured vessels to maintain the integrity of the vascular system, especially via endothelial regeneration. Low EPC levels have been shown to be an independent risk factor for future cardiovascular events and overall mortality in different populations, even after adjustment for diabetes, age and blood glucose. Compared to people without diabetes, those with diabetes have fewer EPCs and impaired EPC function, which may contribute to an increased risk of cardiovascular disease and other vascular complications $[1,2]$.

Aerobic exercise was reported to improve the proliferation, migration and angiogenesis of EPCs in healthy individuals and patients with metabolic syndrome, congestive heart failure, coronary heart disease and prediabetes [37], resulting in maintenance of the endothelial monolayer integrity. The integrity of the endothelial monolayer may play a role in preventing thrombotic complications and atherosclerotic lesion development [8]. Resistance exercise was also reported to improve metabolic profiles in patients with diabetes. A systematic review noted that resistance exercise may be more feasible than aerobic exercise for non-alcoholic fatty liver disease patients with poor cardiorespiratory fitness or for those who cannot tolerate or participate in aerobic exercise [9]. Recently, resistance exercise and combined aerobic and resistance exercise have received increased attention due to their ability to increase the number of EPCs in the peripheral circulation of healthy people [10-13].

The underlying mechanisms of exercise-induced EPC function have not been identified; however, caveolin-1 was found to be abundant in endothelial cells. A previous study showed that caveolin-1 plays a central role in EPC mobilization and homing in stromal cell-derived factor 1 (SDF-1)-driven post-ischaemic vasculogenesis [14]. The increase in EPC function stimulated by PI3K/ AKT could be decreased by a PI3K inhibitor $[15,16]$.

Since little is currently known about the effects of exercise on EPC function, we aimed to explore the effects of aerobic training (AT), resistance training (RT) or a combination of aerobic and resistance training $(\mathrm{AT}+\mathrm{RT})$ on the function of EPCs and the protein expression of caveolin-1, PI3K and AKT in mice with type 2 diabetes. We aimed to provide a helpful reference for the selection of exercise methods to prevent diabetes-related cardiovascular diseases.

\section{Methods}

All animal procedures were performed at Guangxi Medical University. Ethical approval was granted by the Guangxi Medical University Institutional Animal Care and Use Committee. Eight-week-old male type 2 diabetic $\mathrm{db} / \mathrm{db}$ (BKS-Dock Lepr ${ }^{\mathrm{em} 2 \mathrm{Cd} 479} / \mathrm{Nju}$ ) littermates [17] were obtained from the Nanjing Biomedical Research Institute of Nanjing University, Nanjing, China. The mice were randomly divided into the control group, AT group, RT group and AT+RT group ( $n=5 /$ each group). During the 14 days of the intervention, a tail blood sample was obtained for glucose measurement with a glucose metre (Johnson \& Johnson, Milpitas, CA, USA) before starting the experiment and $24 \mathrm{~h}$ after training, which was repeated every week. Type 2 diabetes was diagnosed when the blood glucose level of a mouse was above $11.1 \mathrm{mmol} / \mathrm{l}$ twice. The $\mathrm{db} / \mathrm{db}$ mice had mutant copies of the gene encoding the leptin receptor and exhibited an obese type 2 diabetic phenotype when they were 6 weeks old. Mice were euthanized with intraperitoneal administration of $100 \mathrm{mg} / \mathrm{kg}$ sodium pentobarbital, followed by isolation of the bone marrow-derived EPCs.

\section{Exercise training}

Four or five mice were housed per cage, fed ordinary chow and maintained under a 12-h light/12-h dark cycle. The mice in the control group were maintained in a sedentary state, and the mice in the exercise groups performed AT, RT or AT+RT.

AT was performed on a treadmill (WDW-1, Beijing North Rui Future Analytical Instrument Co., Ltd., Beijing, China) specifically designed for mice. The mice in the AT group were trained for a total of 14 days over 4 weeks (the mice ran 3-4 days a week). Mice were initially trained at a speed of $10 \mathrm{~m} / \mathrm{min}$ for $10 \mathrm{~min} 3$ days/ week in the first week. From the second week, the exercising mice performed AT for $50 \mathrm{~min}$ at a speed of 13$17 \mathrm{~m} / \mathrm{min} 3$ days in the second week and 4 days in the next 2 weeks [18]. All mice ran at the same speed, which increased from 13 to $17 \mathrm{~m} / \mathrm{min}$ when the mice were able to run for $50 \mathrm{~min}$ without rest. The running speed and distance run on the treadmill could be adjusted by the researchers, and thus, we could control the running protocol and training times of the mice.

The mice in the RT group climbed a 1-m custommade ladder composed of a $2-\mathrm{cm}$ grid inclined at $85^{\circ}$ (see Fig. 1 below). The mice in the RT group were trained for a total of 14 days over 4 weeks (the mice ran 3-4 days a week). The mice climbed from the bottom of the ladder to the top while the appropriate weights were attached to their tails. The weight was $50 \%$ of the mouse's body weight in the first week and increased gradually throughout the training period. The mice were motivated to climb the ladder by a gentle pat on their body until they finished three sets of five repetitions of climbing for 3 or 4 days/week for 14 days. The mice were allowed to rest for $1 \mathrm{~min}$ between repetitions and $2 \mathrm{~min}$ between the sets [18]. 


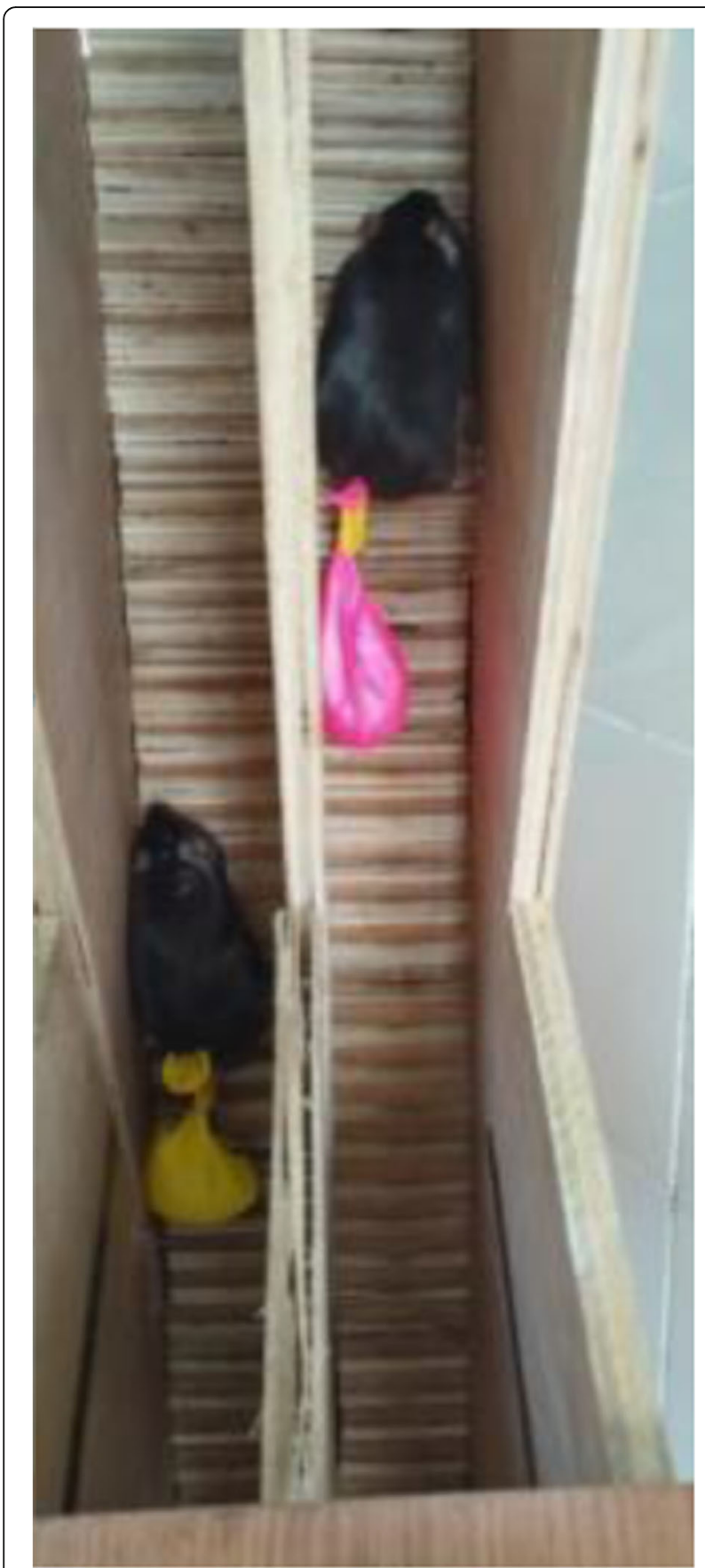

Fig. 1 Image of the ladder. The mice in the AT+RT group performed AT following the protocol of the AT group on Monday, Wednesday, Friday and Sunday and performed RT following the protocol of the RT group on Tuesday, Thursday, Saturday and Sunday. The exercise protocol was discontinued $48 \mathrm{~h}$ before sacrifice

\section{Isolation and culture of bone marrow-derived EPCs}

EPCs were isolated when the exercise protocol was discontinued for $48 \mathrm{~h}$. Five mice from each group (20 mice in total) were intraperitoneally euthanized with $100 \mathrm{mg} / \mathrm{kg}$ sodium pentobarbital in accordance with the rules of the
Institutional Ethical Board for Experimental Procedures. The tibiae and femurs of the mice were bluntly dissected. Then, the medullar channels were flushed with $10 \mathrm{ml}$ of phosphate-buffered saline (PBS, Beijing Solarbio Science \& Technology Co., Ltd., Beijing, China). The cell suspensions were collected by filtration and added to the lymphocyte separating solution (Beijing Solarbio Science \& Technology Co., Ltd., Beijing, China) at a ratio of 1:1. The cell suspensions were centrifuged at $400 \mathrm{~g}$ for $30 \mathrm{~min}$. The cloudy layer of cells was collected and mixed with endothelial cell basal medium-2 (EBM-2; Lonza Group, Ltd., Basel, Switzerland) and then centrifuged at $400 \mathrm{~g}$ for $30 \mathrm{~min}$. The precipitation was subsequently resuspended in EBM-2 containing 10\% foetal bovine serum (FBS; Gibco; Thermo Fisher Scientific, Inc., Waltham, MA, USA) and vascular endothelial growth factor (VEGF; R\&D Systems, Shanghai Sixin Biotechnology Co., Ltd., Shanghai, China). Cells were counted under a microscope and seeded in $25-\mathrm{cm}^{2}$ cell culture bottles coated with human plasma fibronectin purified protein (FPP; R\&D Systems, Shanghai Sixin Biotechnology Co., Ltd., Shanghai, China) at a concentration of $10^{6}$ cells $/ \mathrm{cm}^{2}$. The cells were grown for 4 days. Then, the cells suspended in the medium were removed, and the cells adhering to the surface were cultured for another 3 days [19].

\section{Cellular staining}

Fluorescent chemical detection of EPCs was performed on the attached mononuclear cells after 7 days of culture. Direct fluorescence staining was used to detect the dual binding of fluorescein isothiocyanate (FITC)-labelled Ulex europaeus agglutinin (UEA)-1 (Sigma Chemical Co., St. Louis, MO, USA) and 1,1-dioctadecyl-3,3,3,3-tetramethylindocarbocyanine (DiI)-labelled acetylated low-density lipoprotein (ac-LDL; Molecular Probes, Eugene, OR, USA). Cells were first incubated with ac-LDL $(10 \mu \mathrm{g} / \mathrm{ml})$ at $37^{\circ} \mathrm{C}$ for $4-6 \mathrm{~h}$ and then fixed with $4 \%$ paraformaldehyde for $10 \mathrm{~min}$. Subsequently, the washed cells were incubated with UEA-1 $(10 \mu \mathrm{g} / \mathrm{ml})$ for $1.5 \mathrm{~h}$. After staining, the samples were observed under an inverted fluorescence microscope (IX71, Olympus Corporation, Shibuya, Tokyo, Japan) and further analysed by a laser scanning confocal microscope (FV1000, Olympus Corporation). The cells showing double-positive fluorescence were identified as differentiating EPCs. The EPC number was determined by counting the number of cells in five randomly selected high-power fields $(\times 200)$ via an inverted fluorescence microscope (IX71, Olympus Corporation, Shibuya, Tokyo, Japan). Each experiment was performed with three replicates to ensure the reliability of the data.

\section{Immunofluorescence staining analysis}

The cells were washed with PBS, fixed with $3-4 \mathrm{ml}$ of $4 \%$ paraformaldehyde for $30 \mathrm{~min}$ at $4{ }^{\circ} \mathrm{C}$, washed with 
PBS three times and finally permeabilized with a mixture of $10 \%$ goat serum (Beijing Solarbio Science \& Technology Co., Ltd., Beijing, China) and $0.5 \%$ Triton X-100 (Beijing Solarbio Science \& Technology Co., Ltd., Beijing, China). The cells were immunofluorescently stained with CD31 (Proteintech Group, Inc., Chicago, USA), CD34 (Beijing Bioss Molecular Co., Ltd., Beijing, China), CD133 (Proteintech Group, Inc., Chicago, USA), CD144 (Beijing Bioss Molecular Co., Ltd., Beijing, China) and VEGFR2 (Proteintech Group, Inc., Chicago, USA) antibodies, which were diluted to $100 \mathrm{ml}$, overnight at $4{ }^{\circ} \mathrm{C}$. The cells were then washed with PBS three times (5 min/time) and incubated with CoraLite 488conjugated secondary antibody (Proteintech Group, Inc., Chicago, USA) and Alexa Fluor 594-conjugated secondary antibody (Proteintech Group, Inc., Chicago, USA) in the dark at room temperature for $2 \mathrm{~h}$. The cell DNA was stained with 4',6-diamidino-2-phenylindole (DAPI) (Beijing Solarbio Science \& Technology Co., Ltd., Beijing, China). The cells were examined by fluorescence microscopy (IX71, Olympus Corporation, Shibuya, Tokyo, Japan).

\section{Proliferation assay}

Suspended EPCs were plated on a collagen-coated 96well plate $\left(3.6-4.0 \times 10^{3}\right.$ cells/well) and cultured for $24 \mathrm{~h}$. Subsequently, the suspended EPCs were incubated for another $4 \mathrm{~h}$ in the dark following the addition of $10 \mu \mathrm{l}$ of CCK-8 solution (Dojindo Molecular Technologies, Inc., Kumamoto, Japan) in each well. Then, the plate was agitated for $10 \mathrm{~s}$ to prepare for optical density (OD) measurement, which was performed at an absorbance of $450 \mathrm{~nm}$ with a microplate reader (ELX800; BioTek Instruments, Inc., Winooski, VT, USA).

\section{Adherence assay}

Suspended EPCs were plated on an FPP-coated 96-well plate $\left(2 \times 10^{5} / \mathrm{ml}\right)$ cultured for $24 \mathrm{~h}$ at $4{ }^{\circ} \mathrm{C}$. Subsequently, the suspended EPCs were incubated with CCK-8 solution (Dojindo Molecular Technologies, Inc., Kumamoto, Japan) in each well for another $2 \mathrm{~h}$ in the dark. Then, the plate was agitated for $10 \mathrm{~s}$ to prepare for OD measurement, which was performed at an absorbance of $450 \mathrm{~nm}$ with a microplate reader (ELX800; BioTek Instruments, Inc., Winooski, VT, USA).

\section{Western blot analysis}

After cultivation for 7 days, fractionation of EPCs was performed with RIPA lysis buffer (Beyotime Institute of Biotechnology, Shanghai, China), which contained 1\% phenylmethanesulfonyl fluoride and phosphatase inhibitor (Beijing Solarbio Science \& Technology Co., Ltd., Beijing, China). The protein concentrations were determined by the bicinchoninic acid method according to the manufacturer's instructions (Beyotime Institute of Biotechnology, Shanghai, China). Total protein was separated via $10 \%$ SDS-PAGE, transferred to a polyvinylidene difluoride membrane (Millipore) and blocked with $5 \%$ skim milk. Primary antibodies against the following proteins were used for western blotting: caveolin-1 (Cell Signaling Technology), total PI3K p85 (t-p-PI3Kp85, Abcam), phosphorylated PI3K p85 (p-PI3Kp85, Abcam), total AKT (t-AKT, Cell Signaling Technology) and phosphorylated AKT (p-AKT, Cell Signaling Technology). The membranes were washed with TBST buffer (Beijing Leagene Biotechnology Co., Ltd.) and probed with HRPconjugated secondary antibodies (goat anti-rabbit or goat anti-mouse IgG). Protein band and densitometric analyses were performed using enhanced chemiluminescence (LI-COR Biosciences) to quantify the protein concentrations.

\section{Knockdown of caveolin-1}

EPCs were plated on a 6 -well plate $\left(1 \times 10^{5}\right.$ cells/well $)$ and cultured for $24 \mathrm{~h}$ until the cell confluence was approximately $70-80 \%$. Subsequently, the suspended EPCs were divided into the Lenti-caveolin-1 siRNA silence group (caveolin-1 siRNA) and the Lenti-caveolin-1 siRNA-Mut group (caveolin-1-Mut). The intended meaning of the sentence"EPCs in the caveolin-1 siRNA were incubated a lentiviral vector expressing a caveolin1 small interfering RNA (si) RNA (Shanghai GeneChem Co., Ltd) with a multiplicity of infection (MOI) of 50 . EPCs in caveolin-1-Mut were transfected with a lentiviral vector expressing a caveolin-1 non-targeting RNA (Shanghai GeneChem Co., Ltd). Polybrene with $8 \mu \mathrm{g} / \mathrm{ml}$ was added to accelerate transfection. Transfection was performed at $37^{\circ} \mathrm{C}$ for $6 \mathrm{~h}$. Subsequently, the cells were photographed after culture for another $72 \mathrm{~h}$.

\section{Statistical analysis}

All variables were tested for normality by the Kolmogorov-Smirnov test. Levene's test was performed to test for equality of variances. If the variables were homogeneous, one-way ANOVA was used for the analysis of the different variables among the groups, and the Tukey test was used for post hoc analyses; otherwise, the Welch test was chosen for alternative between-group comparisons, and the Tamhane test was used for post hoc analyses. Data are shown as the mean \pm SE. $P<0.05$ (two-tailed) was defined as significant. Data were analysed using SPSS software 22.0 (SPSS Inc., Chicago, IL, USA).

\section{Results}

\section{Characterization of EPCs}

As shown in Fig. 2, after 4 days of growth in vitro, most of the EPCs were round or oval, and a few were spindleshaped. On the 8th day, the EPCs were mostly spindle- 


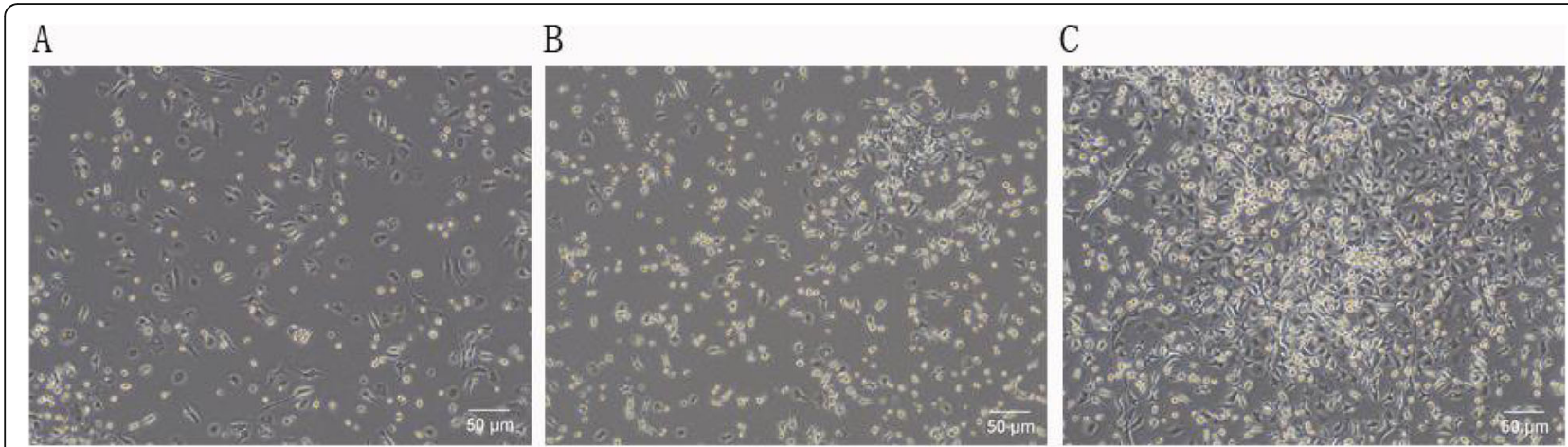

Fig. 2 Characterization of EPCs of mice isolated from the control group growing in vitro (magnification $\times 100$ ). a Round or oval EPCs on the 4th day. b Spindle-shaped or round EPCs on the 8th day. c EPCs covering the bottom, similar to "stone pavement", on the 14th day. After 7 days of growth, the bone marrow-derived EPCs from mice were characterized as adherent cells that showed Dil-ac-LDL uptake and lectin binding via laser scanning confocal microscopy (Fig. 3). A total of $73.50 \pm 7.47 \%$ of the adherent cells showed Dil-ac-LDL uptake and lectin binding after growing for 7 days. In addition, these findings were confirmed by the expression of the well-established cell surface markers CD31, CD34, CD133, CD144 and VEGFR2, which were detected in $91.2 \pm 3.6 \%, 95.3 \pm 2.6 \%, 94.8 \pm 3.2 \%, 93.1 \pm 4.2 \%$ and $95.8 \pm 2.5 \%$ of all cells by immunofluorescence staining (see details in Fig. 4 )

shaped and round. On the 14th day, the cells grew well and adhered to the bottom of the culture bottle, similar to "stone pavement" (Figs. 3 and 4).

\section{Changes in glucose between the groups with different interventions}

After interventions for 14 days, the blood glucose of mice in the RT and $\mathrm{AT}+\mathrm{RT}$ groups was significantly lower than that in the control group (see Fig. 5 for more details).

\section{Proliferative ability}

As shown in Fig. 6a, cell proliferation increased in the AT, $\mathrm{RT}$ and AT+RT groups compared with the control group after 14 days of intervention. The proliferative activities of the EPCs were significantly lower in the control group than in the AT group (vs control: $95 \% \mathrm{CI}-0.49$ to approximately $-0.14, P<0.001$ ), the RT group (vs control: $95 \% \mathrm{CI}-0.38$ to approximately $-0.04, P=0.015$ ) and the AT+RT group (vs control: $95 \% \mathrm{CI}-0.46$ to approximately $-0.12, P=0.001$ ). There were no significant differences in the proliferative activity among the AT, RT and AT+RT groups. Knockdown of caveolin-1 retarded EPC growth. An increased proliferative capability was found in the control group compared to the caveolin-1-siRNA-silenced group (vs control: 95\% CI 0.19 0.30, $P<0.001$ ), but no difference was found between the control group and the negative control group (the caveolin-1-siRNA Mut group).

\section{Adherent ability}

Exercise induced EPC adherence during the intervention. Compared with the control group, the AT+RT group showed significant increases in EPC adherence (vs control: $95 \% \mathrm{CI}-0.39$ to approximately $-0.12, P<0.001$ ), and this increase was more notable in the $\mathrm{AT}+\mathrm{RT}$ group than in the AT (vs AT+RT: 95\% CI 0.03 0.30, $P=0.008$ ) or RT (vs AT+RT: 95\% CI 0.04 0.32, $P=0.017$ ) groups. Caveolin-1 deficiency inhibited EPC adherence, which was 30\% lower than that of the control group (vs control: 95\% CI 0.17 0.51, $P=0.002)$.

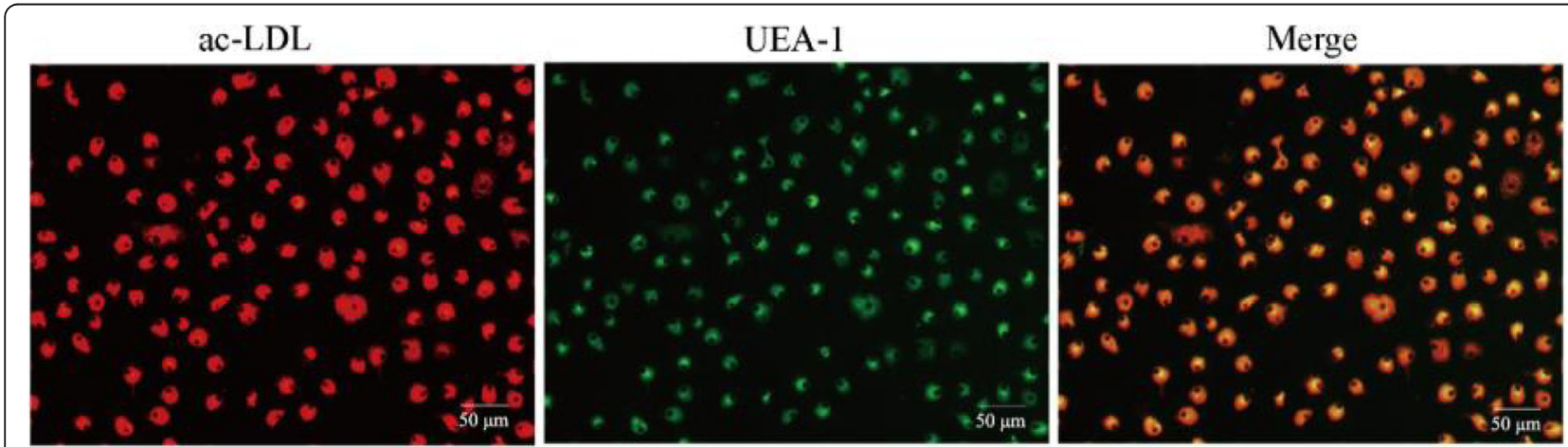

Fig. 3 Bone marrow-derived EPCs of mice isolated from the control group after 7 days of growth. Dil-ac-LDL uptake (red) and lectin binding (green) of adherent cells were identified via laser scanning confocal microscopy. Double-positive cells appearing yellow in the overlay were identified as differentiating EPCS (magnification $\times 200$ ) 


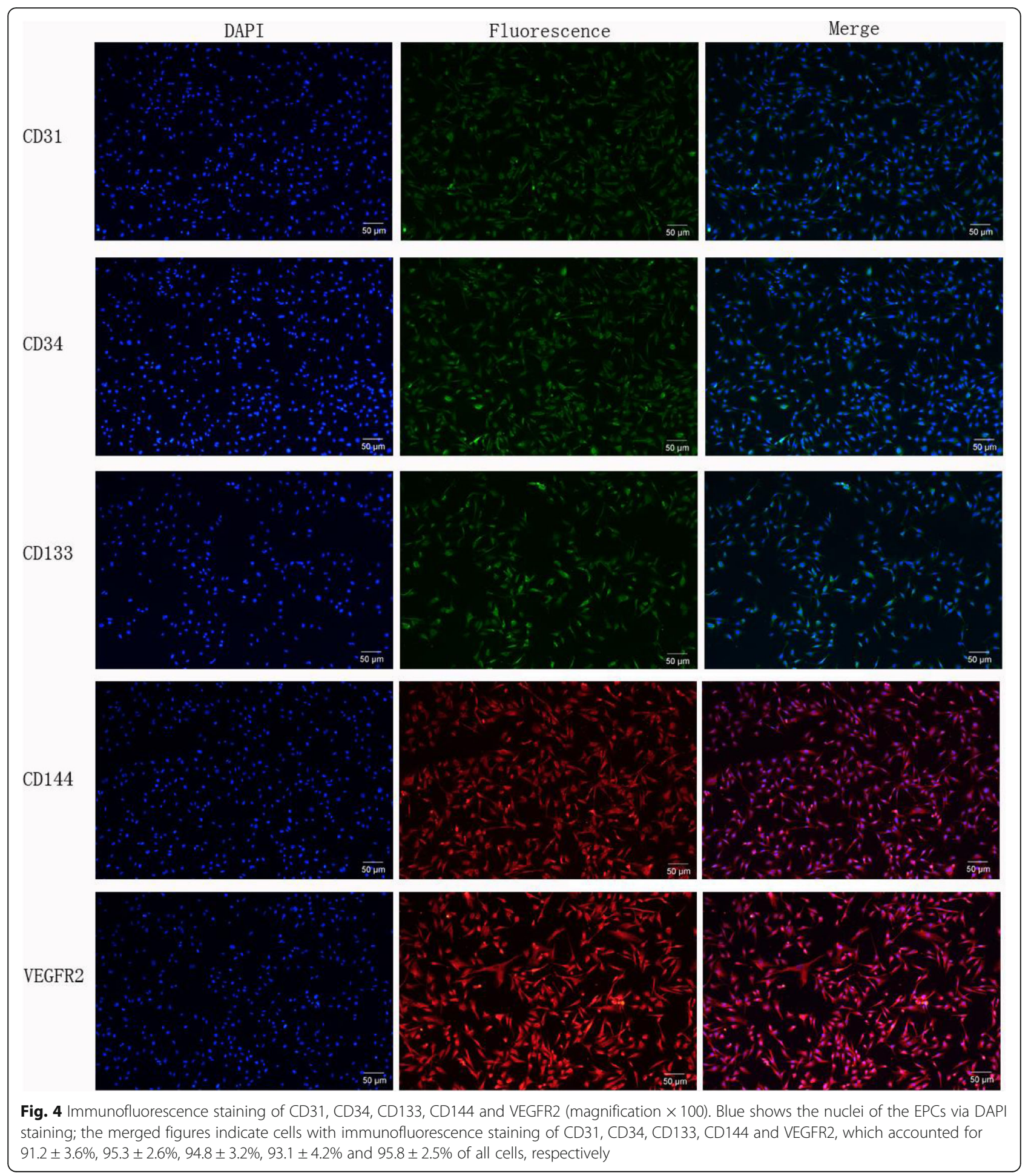

The concentration of caveolin-1 and PI3K/AKT determined by western blot analysis

Figure 7 showed that the AT (vs control: $95 \% \mathrm{CI}-2.17$ to approximately $-1.28, P<0.001$ ), RT (vs control: $95 \% \mathrm{CI}-$ 2.23 to approximately $-1.34, P<0.001$ ) and AT + RT (vs control: $95 \% \mathrm{CI}-2.33$ to approximately $-1.44, P<0.001$ ) groups exhibited significant increases in caveolin-1. There was a clear difference in the levels of p-PI3K (95\% CI 0.79 to approximately $-0.09, P=0.012)$ and $\mathrm{p}$-AKT $(95 \%$ CI -0.72 to approximately $-0.03, P=0.030$ ) between the $\mathrm{AT}+\mathrm{RT}$ and control groups. To elucidate the mechanism by which AT+RT improved EPC function, we investigated 


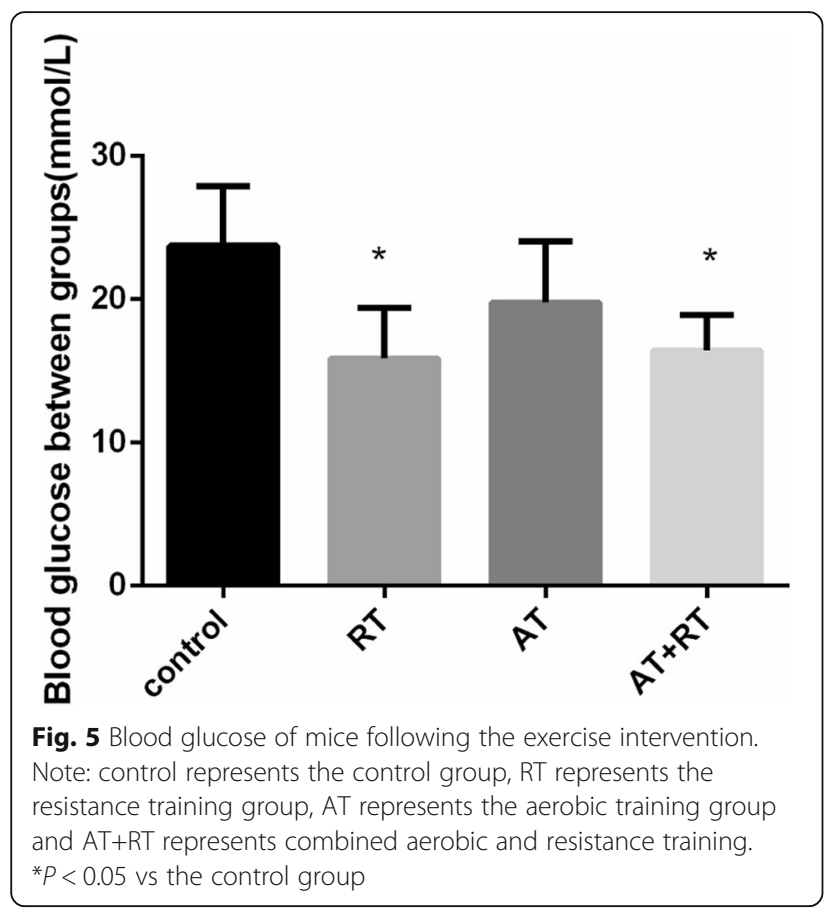

the effects of caveolin-1 knockdown on EPC p-PI3K and $\mathrm{p}-\mathrm{AKT}$ protein expression. AT+RT contributed to a notable increase in the EPC p-PI3K and p-AKT protein levels, which were reduced in caveolin-1 knockdown EPCs (see Fig. 8 for details).

\section{Discussion}

This study explored the effect of 14-day RT, AT and combination training on the function of EPCs and the potential mechanism in mice with type 2 diabetes.
The data revealed that (i) RT, AT and a combination of both can significantly improve the proliferative functions of EPCs in mice with type 2 diabetes and that $\mathrm{AT}+\mathrm{RT}$ induced the greatest improvement in EPC adherence; (ii) $\mathrm{AT}+\mathrm{RT}$ induced increases in caveolin-1 and PI3K AKT; and (iii) knockdown of caveolin-1 abolished the positive effect of AT+RT on the functions of EPCs and protein expression (caveolin-1, PI3K, AKT).

Diabetes impairs the angiogenic ability of EPCs, which significantly enhances the risk of atherosclerotic disease and increases cardiovascular morbidity and mortality [2]. AT has been well studied and shown to enhance the function of EPCs in patients with hypertension, obesity, metabolic syndrome, coronary artery disease and chronic heart failure [3-7]. However, the effect of AT, RT or $\mathrm{AT}+\mathrm{RT}$ on the function of EPCs in people or animal models with type 2 diabetes has not yet been explored.

Our data suggest that exercise (AT, RT and AT+RT) inhibited EPC proliferation. A single session of RT was reported to stimulate SDF- $1 \alpha$, VEGF, hypoxia-inducible factor 1- $\alpha$ (HIF-1 $\alpha)$ and matrix metalloproteinase-9 [10-12], which were shown to enhance the proliferative, migratory and angiogenic abilities of EPCs [20-22]. These studies support our finding that RT enhanced EPC proliferative activity in mice with type 2 diabetes. RT or RT combined with AT was documented to increase the number of EPCs in healthy volunteers [10-13]. Our study indicated that an increase in EPC number may contribute to the enhanced EPC proliferative capability after RT or AT + RT.

The positive effects of AT or RT were combined in AT+RT. Mice in the AT+RT group showed especially notable EPC adherence. The AT+RT intervention resulted in the greatest improvements in blood glucose, plasma lipids and fasting insulin in patients

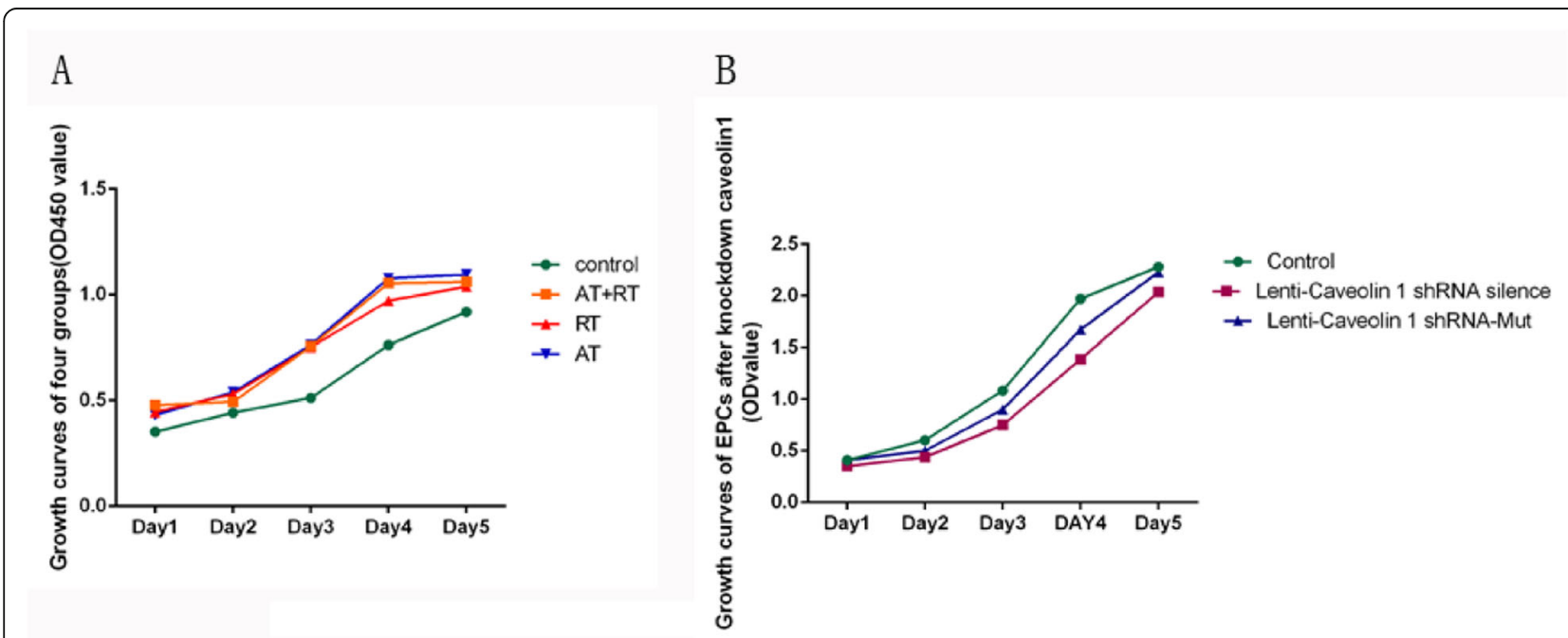

Fig. 6 Growth curve of the EPCs. a Growth curves of the EPCs after 14 days of intervention. $\mathbf{b}$ Growth curves of the EPCs after knockdown of caveolin-1. AT+RT, combined aerobic and resistance training; RT, resistance training; AT, aerobic training 


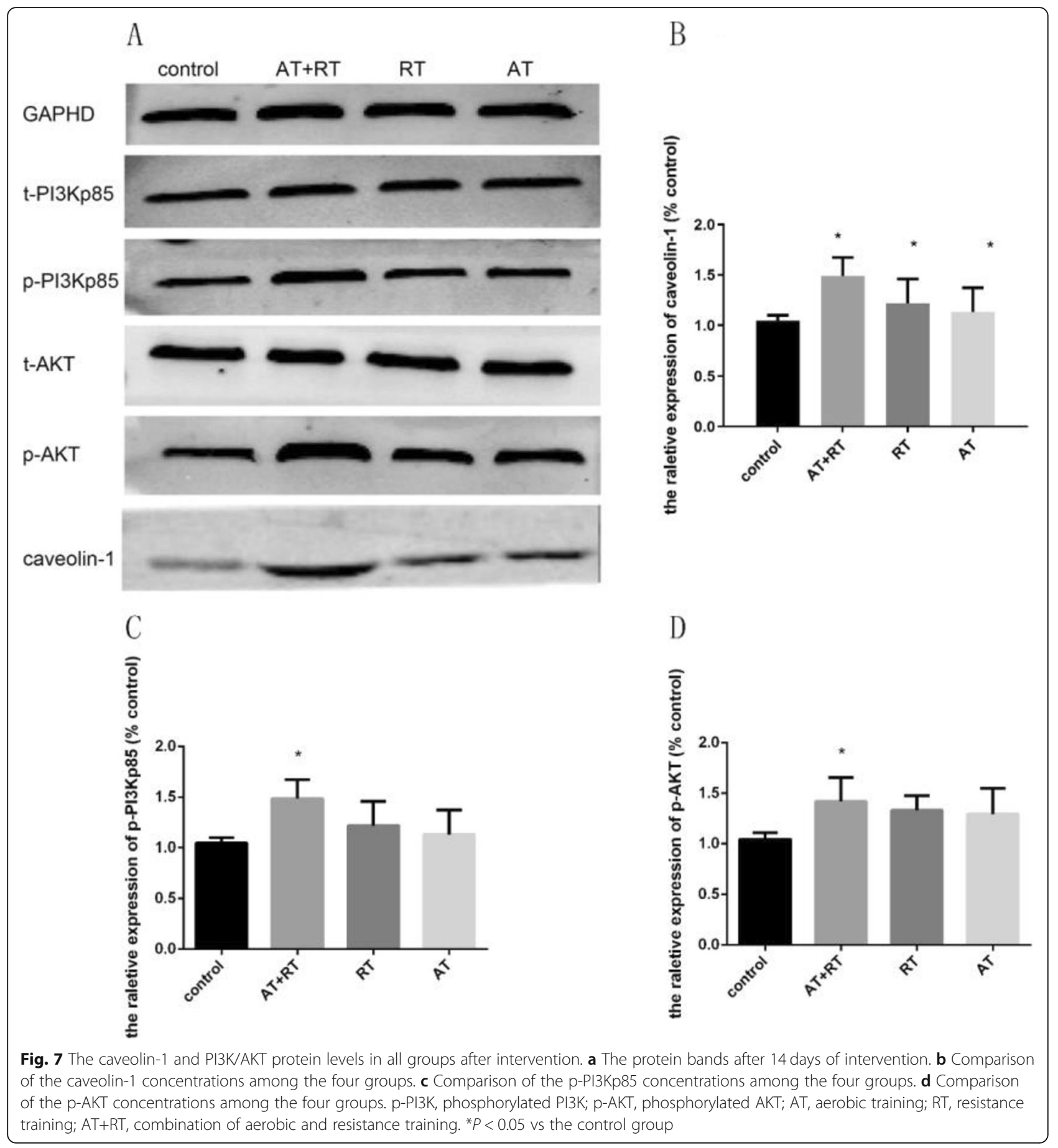

with and animal models of type 2 diabetes [23]. Previous studies reported the effect of $\mathrm{AT}, \mathrm{RT}$ and $\mathrm{AT}+\mathrm{RT}$ on insulin resistance (HOMA-IR) in patients with high blood glucose, in which the AT+RT resulted in the maximum improvement of HOMA-IR [24], thus alleviating the negative effects of high blood glucose on EPCs. Decreased numbers and dysfunction of EPCs have been shown to be independent risk factors for future cardiovascular events and overall mortality in different populations. Through reversing the diabetes-mediated dysfunction of EPCs, combined aerobic and resistance training may be a possible strategy for the prevention of diabetes-related cardiovascular diseases.

We observed an increase in the protein expression of caveolin-1 after exercise. To further elucidate the mechanism underlying exercise-induced improvement of EPC proliferation and adherence, we explored the impact of 


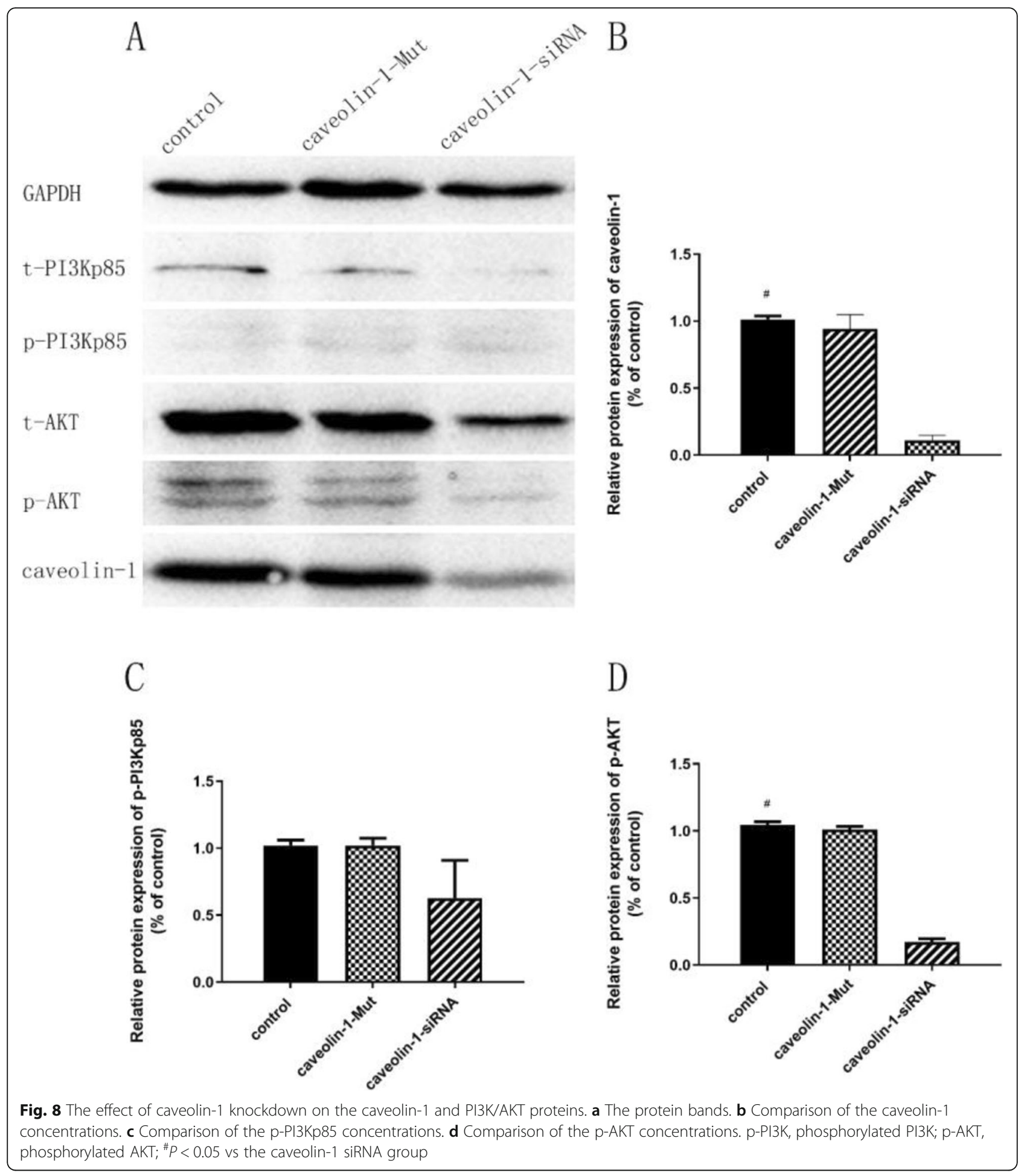

caveolin-1 on EPC function by cellular transfection of a lentiviral vector with caveolin-1-siRNA. We found that reduced expression of caveolin-1 and inhibition of EPC functions were detected in EPCs infected with caveolin-1-siRNA. Caveolin-1 is involved in the regulation of transcytosis, permeability, vascular tone and angiogenesis. One study showed that knockdown of caveolin-1 inhibited endothelial cell function [25]. A previous study suggested that treadmill exercise can enhance EPC function via a significant increase in the concentration of caveolin-1 [26]. Consistent with these two studies, our study indicated that AT+RT 
enhanced EPC function by upregulating caveolin-1 in mice with type 2 diabetes.

Our study showed that exercise promoted EPC function and increased p-PI3K and p-AKT, which were abolished by knockdown of caveolin-1. PI3K/AKT was reported to promote EPC function. Another study demonstrated that the increase in PI3K/AKT induced by exercise can lead to further enhancement of the function of EPCs [27], which was consistent with our study. A previous report demonstrated that the modulation of caveolin-1 expression can affect signalling through the PI3K/AKT pathway and cellular proliferation [28]. AKT expression was downregulated in embryonic fibroblast cells in caveolin-1 knockout mice, resulting in inhibited cell functions [29], which was consistent with our study.

Caveolin-1 has been investigated by a series of studies providing insight into the confusing effects of caveolin-1 on cellular function. In an article published in Circulation, we found that endothelial cell function was significantly abrogated in mice without the caveolin-1 gene [30]. Another study also provided similar evidence: caveolin-1 knockdown via caveolin-1 siRNA was reported to decrease $\mathrm{p}-\mathrm{AKT}$, resulting in reduced cell proliferation [31]. These two studies provide support for the results of our study. When AKT was activated, p-AKT colocalized with caveolin-1 in human bone marrowderived mesenchymal stem cells exhibiting osteogenic differentiation [32]. Based on these findings and previous studies of caveolin-1, we hypothesized that the exercise induces alterations invascular shear stress by increasing blood flow and tissue fluid flow [20,33], which rises the level of vascular endothelial growth factor [16] to trigger an increase of phosphorylated PI3K/AKT by caveolin1 $[31,34]$. Caveolin1 through compartmentalization of VEGF receptor with VEGF provides a structural platform to facilitate and amplifies signalling cascades like the PI3K/AKT pathway $[31,35]$, resulting in increasing the bioactivities of adhesion and proliferation of EPC $[16,35]$.

The limitations of this study include the fact that in vitro observations cannot indicate the impact on the entire body, the short time period of the intervention and the lack of baseline variables regarding EPC function. However, a control group with no specific exercise intervention was used to identify the effects of exercise and remaining sedentary on EPC function in this randomized controlled study.

\section{Conclusion}

Our study explores the effect of 14-day RT, AT and combination training on the function of EPCs and the potential mechanism in mice with type 2 diabetes. The data revealed that $\mathrm{AT}+\mathrm{RT}$ induced the greatest improvement in adherence of EPCs and increases in caveolin-1 and PI3K AKT; knockdown of caveolin-1 abolished the positive effect of AT+RT on the functions of EPCs and protein expression (caveolin-1, PI3K AKT). Our study will provide a helpful reference for the selection of exercise methods and promote further development of novel cell-based therapeutic strategies, resulting in improved management of diabetes-related cardiovascular diseases.

\begin{abstract}
Abbreviations
AT: Aerobic training; RT: Resistance training; AT+RT: Combined aerobic and resistance training; EPCs: Endothelial progenitor cells; Caveolin-1-

siRNA: Caveolin-1 small interfering RNA; SDF-1: Stromal cell-derived factor 1; PBS : Phosphate-buffered saline; EBM-2: Endothelial cell basal medium-2; FBS: Foetal bovine serum; VEGF: Vascular endothelial growth factor; FPP: Fibronectin purified protein; FITC: Fluorescein isothiocyanate; UEA: Ulex europaeus agglutinin; Dil: Tetramethylindocarbocyanine; ac-LDL: Acetylated low-density lipoprotein; DAPI: 4',6-Diamidino-2-phenylindole; OD: Optical density; t-p-PI3Kp85: Total PI3K p85; p-PI3Kp85: Phosphorylated PI3K p85; tAKT: Total AKT; p-AKT: Phosphorylated AKT; HIF-1a: Hypoxia-inducible factor 1-a; HOMA-IR: Homeostasis model assessment insulin resistance; MOI: Multiplicity of infection
\end{abstract}

\section{Acknowledgements}

Not applicable

\section{Authors' contributions}

All authors made contributions to this study by designing the study (XD, FH), planning the experiments (LZ, WPZ, QYC, CM, WW), performing the cellular experiments (WPZ, YHL, TG, ZCL, YFH), analysing and interpreting the data $(L Z, Y H L)$, drafting the manuscript $(L Z, Y H L)$ and reviewing and editing the manuscript (XD, FH). All authors critically reviewed the manuscript and read and approved the final version.

\section{Funding}

This work was supported by the National Natural and Scientific Foundation (81660147, 81760057).

\section{Availability of data and materials}

The datasets analysed during the current study are available from the corresponding authors on reasonable request.

\section{Ethics approval and consent to participate}

Eight-week-old male type 2 diabetic $\mathrm{db} / \mathrm{db}$ were used in this study. Ethical approval was granted by the Guangxi Medical University of Institutional Animal Care and Use Committee.

\section{Consent for publication}

Not applicable.

\section{Competing interests}

The authors declare that they have no competing interests.

\section{Author details}

1Department of Endocrinology, The First Affiliated Hospital of Guangxi Medical University, Nanning 530021, China. ${ }^{2}$ Department of Nursing, Guangxi JiangBin Hospital, Nanning 530021, China. ${ }^{3}$ Department of Cardiology, The First Affiliated Hospital of Guangxi Medical University, Nanning 530021, China. ${ }^{4}$ Department of Gastroenterology, The First Affiliated Hospital of Guangxi Medical University, Nanning 530021, China. ${ }^{5}$ Department of Internal Medicine, The First Affiliated Hospital of Guangxi Medical University, Nanning 530021, China.

Received: 24 May 2019 Revised: 26 November 2019

Accepted: 10 December 2019 Published online: 03 January 2020

\section{References}

1. Rigato M, Bittante C, Albiero M, Avogaro A, Fadini GP. Circulating progenitor cell count predicts microvascular outcomes in type 2 diabetic patients. J Clin Endocrinol Metab. 2015;100(7):2666-72.

2. Schmidt-Lucke C, Rössig L, Fichtlscherer S, et al. Reduced number of circulating endothelial progenitor cells predicts future cardiovascular events: 
proof of concept for the clinical importance of endogenous vascular repair. Circulation. 2005;111(22):2981-7.

3. Tsai H-H, Lin C-P, Lin Y-H, Hsu C-C, Wang J-S. High-intensity interval training enhances mobilization/functionality of endothelial progenitor cells and depressed shedding of vascular endothelial cells undergoing hypoxia. Eur J Appl Physiol. 2016;116(11-12):2375-88.

4. Sonnenschein $\mathrm{K}$, Horváth $\mathrm{T}$, Mueller M, et al. Exercise training improves in vivo endothelial repair capacity of early endothelial progenitor cells in subjects with metabolic syndrome. Eur J Cardiovasc Prev Rehabil. 2011;18(3):406-14.

5. Sandri M, Viehmann $M$, Adams V, et al. Chronic heart failure and agingeffects of exercise training on endothelial function and mechanisms of endothelial regeneration: results from the Leipzig Exercise Intervention in Chronic heart failure and Aging (LEICA) study. Eur J Prev Cardiol. 2016;23(4): $349-58$

6. Sen S, Islam A, Witkowski S. Changes in function and gene expression in endothelial progenitor cells (EPCS) leads to improvement in flow mediated dilatation and serum markers in patients with prediabetes following aerobic exercise. Paper presented at: DIABETES2014.

7. Hoellriegel $\mathrm{R}$, Beck E, Woitek F, et al. Regular physical exercise training partially corrects metabolic alterations and improves peripheral endothelial function in pre-diabetic, adipose patients with coronary artery disease. Paper presented at: American Journal of Respiratory and Critical Care Medicine 2011.

8. Urbich C, Dimmeler S. Endothelial progenitor cells: characterization and role in vascular biology. Circ Res. 2004;95(4):343-53.

9. Hashida R, Kawaguchi T, Bekki M, et al. Aerobic vs. resistance exercise in nonalcoholic fatty liver disease: a systematic review. J Hepatol. 2017;66(1):142-52.

10. Ross MD, Wekesa AL, Phelan JP, Harrison M. Resistance exercise increases endothelial progenitor cells and angiogenic factors. Med Sci Sports Exerc. 2014:46(1):16-23.

11. Ribeiro F, Ribeiro IP, Gonçalves AC, et al. Effects of resistance exercise on endothelial progenitor cell mobilization in women. Sci Rep. 2017;7(1):17880.

12. Waclawovsky G, Umpierre D, Figueira FR, et al. Exercise on progenitor cells in healthy subjects and patients with type 1 diabetes. Med Sci Sports Exerc. 2015;48:190-9.

13. Park J-H, Miyashita M, Kwon Y-C, et al. A 12-week after-school physical activity programme improves endothelial cell function in overweight and obese children: a randomised controlled study. BMC Pediatr. 2012;12(1):111.

14. Sbaa E, DeWever J, Martinive P, et al. Caveolin plays a central role in endothelial progenitor cell mobilization and homing in SDF-1-driven postischemic vasculogenesis. Circ Res. 2006;98(9):1219-27.

15. Meng S, Cao J-T, Zhang B, Zhou Q, Shen C-X, Wang C-Q. Downregulation of microRNA-126 in endothelial progenitor cells from diabetes patients, impairs their functional properties, via target gene Spred-1. J Mol Cell Cardiol. 2012;53(1):64-72

16. Obi S, Masuda H, Shizuno T, et al. Fluid shear stress induces differentiation of circulating phenotype endothelial progenitor cells. Am J Phys Cell Phys. 2012;303(6):C595-606.

17. Garris DR. Estrogenic stimulation of ovarian follicular maturation in diabetes (db/db) mutant mice: restoration of euglycemia prevents hyperlipidemic cytoatrophy. Cell Tissue Res. 2004;318(2):365-73.

18. Tuon T, Souza PS, Santos MF, et al. Physical training regulates mitochondrial parameters and neuroinflammatory mechanisms in an experimental model of Parkinson's disease. Oxidative Med Cell Longev. 2015;2015. https://doi. org/10.1155/2015/261809.

19. Guo Y, Peng R, Liu Q, Xu D. Exercise training-induced different improvement profile of endothelial progenitor cells function in mice with or without myocardial infarction. Int J Cardiol. 2016;221:335-41.

20. De Biase C, De Rosa R, Luciano R, et al. Effects of physical activity on endothelial progenitor cells (EPCs). Front Physiol. 2014;4:414.

21. Huang $\mathrm{P}-\mathrm{H}$, Chen $\mathrm{Y}-\mathrm{H}$, Wang $\mathrm{C}-\mathrm{H}$, et al. Matrix metalloproteinase- 9 is essential for ischemia-induced neovascularization by modulating bone marrow-derived endothelial progenitor cells. Arterioscler Thromb Vasc Biol. 2009:29(8):1179-84.

22. Rocha NG, Sales AR, Penedo LA, et al. Impaired circulating angiogenic cells mobilization and metalloproteinase-9 activity after dynamic exercise in early metabolic syndrome. Biomed Res Int. 2015;2015. https://doi.org/10.1155/ 2015/920356.

23. Mann S, Beedie C, Balducci S, et al. Changes in insulin sensitivity in response to different modalities of exercise: a review of the evidence Diabetes Metab Res Rev. 2014;30(4):257-68.
24. AbouAssi H, Slentz CA, Mikus CR, et al. The effects of aerobic, resistance, and combination training on insulin sensitivity and secretion in overweight adults from STRRIDE AT/RT: a randomized trial. J Appl Physiol. 2015;118(12): $1474-82$.

25. Tahir SA, Park S, Thompson TC. Caveolin-1 regulates VEGF-stimulated angiogenic activities in prostate cancer and endothelial cells. Cancer Biol Ther. 2009;8(23):2284-94.

26. Zhao Y, Pang Q, Liu M, et al. Treadmill exercise promotes neurogenesis in ischemic rat brains via caveolin-1/NEGF signaling pathways. Neurochem Res. 2017;42(2):389-97.

27. Cheng XW, Kuzuya M, Kim W, et al. Exercise training stimulates ischemiainduced neovascularization via phosphatidylinositol 3-kinase/Akt-dependent hypoxia-induced factor-1a reactivation in mice of advanced age. Circulation. 2010;122(7):707.

28. Matthews LC, Taggart MJ, Westwood M. Modulation of caveolin-1 expression can affect signalling through the phosphatidylinositol 3-kinase/ Akt pathway and cellular proliferation in response to insulin-like growth factor I. Endocrinology. 2008;149(10):5199-208.

29. Liao W-X, Feng L, Zheng J, D-b C. Deciphering mechanisms controlling placental artery endothelial cell migration stimulated by vascular endothelial growth factor. Endocrinology. 2010;151(7):3432-44.

30. Sonveaux P, Martinive P, DeWever J, et al. Caveolin-1 expression is critical for vascular endothelial growth factor-induced ischemic hindlimb collateralization and nitric oxide-mediated angiogenesis. Circ Res. 2004; 95(2):154-61.

31. Feng L, Liao WX, Luo Q, et al. Caveolin-1 orchestrates fibroblast growth factor 2 signaling control of angiogenesis in placental artery endothelial cell caveolae. J Cell Physiol. 2012;227(6):2480-91.

32. Baker N, Sohn J, Tuan RS. Promotion of human mesenchymal stem cell osteogenesis by PI3-kinase/Akt signaling, and the influence of caveolin-1/ cholesterol homeostasis. Stem Cell Res Ther. 2015;6(1):238.

33. Green DJ, Maiorana A, O'Driscoll G, Taylor R. Effect of exercise training on endothelium-derived nitric oxide function in humans. J Physiol. 2004;561(1): $1-25$.

34. Albinsson S, Nordstrom I, Sward K, Hellstrand P. Differential dependence of stretch and shear stress signaling on caveolin-1 in the vascular wall. Am J Phys Cell Phys. 2008;294(1):C271-9.

35. Luo T, Shu J, Lu Z, Han T, Fang G, Xue X. Potential role of caveolin-1 in regulating the function of endothelial progenitor cells from experimental MODS model. Mediat Inflamm. 2019;2019. https://doi.org/10.1155/2019/ 8297391

\section{Publisher's Note}

Springer Nature remains neutral with regard to jurisdictional claims in published maps and institutional affiliations.

Ready to submit your research? Choose BMC and benefit from:

- fast, convenient online submission

- thorough peer review by experienced researchers in your field

- rapid publication on acceptance

- support for research data, including large and complex data types

- gold Open Access which fosters wider collaboration and increased citations

- maximum visibility for your research: over $100 \mathrm{M}$ website views per year

At BMC, research is always in progress.

Learn more biomedcentral.com/submissions 